\title{
Effect of Marginal Discrepancy induced by CAD/CAM and Conventional Ceramic Processing Techniques in All Ceramic Complete Veneer Retainers - A Systematic Review
}

\author{
${ }^{1}$ Dr.Dhanraj, ${ }^{2}$ Anusha Sathyamurthy.

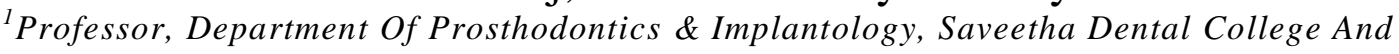 \\ Hospital,Saveetha University, \\ ${ }^{2}$ No:162 Poonamalle High Road, Chennai-600077, Tamilnadu, India.
}

\begin{abstract}
ST TRUCTUREDABSTRACT:
Statement Of The Problem:

Marginal discrepancy of all ceramic complete veneer restorations is dependent mainly on the processing technique employed in the fabrication of the restoration. The comprehensive role of processing technique, inducing the amount of marginal discrepancy needs to be investigated further.
\end{abstract}

Aim:

To evaluate the amount of marginal discrepancy induced by CAD/CAM technique and conventional ceramic processing techniques in all ceramic complete veneer retainers.

Search Methodology:

An electronic search was launched with pubmed, Cochrane, ScienceDirect databases to screen for articles discussing the marginal discrepancy associated with all ceramic complete veneer retainers using suitable keywords. The database search yielded 70 articles out of which, 16 were selected based on the inclusion criteria. The included articles were then subjected to data extraction and subsequent statistical analysis.

\section{Results}

The results of the present systematic review has analysed the marginal discrepancy in all ceramic complete veneer retainers and inferred marginal discrepancy induced by CAD/CAM technique is 55.35 (54.4-65.3)microns at 95 percent confidence interval and conventional techniques recorded marginal discrepancy of 56.53(44.70-68.38) microns at 95 percent confidence interval ( $p<.05)$.

\section{Conclusion}

The marginal discrepancy induced by CAD/CAM and conventional techniques .viz slip casting,copy milling, heat pressing are similar and both the systems can be employed to fabricate all ceramic complete veneer retainers.

Key Words:all ceramics, CADCAM ceramics, conventional ceramics, marginal discrepancy

\section{Introduction}

The marginal fit is one of the most important criteria for long term success of all ceramic restorations. Discrepancy in marginal fit facilitate microleakage salivary infiltration and seepage resulting in dissolution of luting agent, thus increases the susceptibility for secondary caries around abutments eventually leading to abutment and restoration failure. Marginal discrepancy also inflicts severe sensitivity due to the exposure of exposed dentinal tubules. Also marginal discrepancy results in collection of plaque and food particles around the exposed margins which subsequently initiates periodontal breakdown in the abutment teeth.

Various factors like type of finish lines, die spacing, choice of restorative materials and cementation procedure influence this phenomenon.

Amongst these factors cementation procedures influence the marginal seating of all ceramic complete veneer retainers markedly. Among the luting agents, the resin bonded cements possess a greater film thickness more than 25 microns which may interfere with complete marginal seating of the retainers.

The other factors influencing marginal discrepancy include type of ceramic system selected viz: polycrystalline based ceramics, glass ceramics ,glass infiltrated ceramics, castable ceramics, pressable ceramics and feldspathic ceramics. The manufacturing and processing technique sintering, slip casting, heat pressing, casting, CAD/CAM also significantly influence the marginal discrepancy .In addition to this the time interval between the fabrication stages, sequence of firing, the bulk of 
the material added for firing, the core and veneering layer mismatch, type of porcelain - high fusing, medium fusing and low fusing, play a role in influencing marginal discrepancy.

$\mathrm{CAD} / \mathrm{CAM}$ restorations are becoming increasingly popular due to its several advantages over the conventional ceramic processing techniques. Several authors have reported varying levels of marginal discrepancy associated with $\mathrm{CAD} / \mathrm{CAM}$ restorations, This technique offers a great advantage over conventional processing techniques by eliminating the clinical steps in impression making and laboratory steps including cast pouring, articulation, die sectioning , casting and subsequent layering, thus saving enormous amount of time and manpower,but the superiority of this system over the conventional ones with effect to marginal discrepancy is not clearly established in the literature. Hence this systematic review was attempted to study the influence of processing techniques over marginal discrepancy in all ceramic complete veneer retainers.

\section{Null Hypothesis}

Null hypothesis formulated for the review was, the processing technique does not have any influence in inducing marginal discrepancy in all ceramic complete veneer retainers.

\section{Alternate Hypothesis}

Alternate hypothesis formulated for the review was, the processing technique does have influence in inducing marginal discrepancy in all ceramic complete veneer retainers.

\section{A I M:}

To evaluate the amount of marginal discrepancy induced by CAD/CAM technique and conventional ceramic processing techniques in all ceramic complete veneer restorations

\section{1 sources Used:-}

\section{Materials And Methods:-}

An electronic search was conducted for articles written in English, translated into English listed with pubmed,Cochrane,Science Direct till July $1^{\text {st }} 2013$.

The search methodology applied was a combination of MESH terms and keywords included are, Metal ceramic crowns, All ceramic crowns, Porcelain fused metal crowns, Zirconia crowns, Marginal accuracy of metal ceramic crowns, Marginal accuracy of all ceramic crowns, Marginal accuracy of porcelain fused crowns, Marginal accuracy of zirconia crowns, Marginal fit of all ceramic crowns, Marginal fit of acrylic crowns, Marginal fit of zirconia crowns, Marginal fit of porcelain fused crowns, Marginal discrepancy of metal ceramic crowns.Marginal discrepancy of all ceramic crowns, Marginal discrepancy of porcelain fused crowns, Marginal discrepancy of zirconia crowns, All ceramic restorations, All ceramic veneers, All ceramic fixed partial denture, All ceramic crowns, Metal free crowns, All ceramic bridges. Alumina crowns, Zirconia crowns,Lithium disilicate crowns, Dicor crowns, Cerestore crowns, Emax press crowns, Empress crowns, CAD/CAM TECHNIQUE, CAD/CAM crowns, Copymilling technique, Sintered crowns, Luting agents, Luting ceramic crowns, CAD/CAM. Heat pressing, Copy milling, Slip casting, Marginal exposure, Secondary caries.

Review articles as well as references from the different studies were also used to identify the relevant articles. Further the manual search was conducted and additional articles could not be identified.

\section{Selection Of Studies:}

The review process consist of two phases.In the first phase titles and abstract of the search were initially screened for relevance and the full text of relevant abstract were obtained and accessed.The hand search of selected journals as well as search of references in the selected studies were also done. The artIcles that were obtained after first step of review process using the following inclusion and exclusion criteria were screened in second phase and relevant and suitable articles were isolated for further processing and data extraction.

\section{Inclusion Criteria}

The articles discussing the following parameters were included for the systematic review.

a. Randomized controlled trials reporting marginal discrepancy in all ceramic complete veneer retainers.

b. Controlled in-vitro trials reporting marginal discrepancy in all ceramic complete veneer retainers. 
c. Experimental research reporting $\mathrm{CAD} / \mathrm{CAM}$ processing techniques for all ceramic complete veneer retainers.

d. Experimental research reporting heat processed techniques for all ceramic complete veneer retainers.

e. Experimental research reporting injection moulding technique for all ceramic complete veneer retainers.

f. Experimental research reporting casting technique for all ceramic complete veneer retainers.

g. In vitro trials utilizing customized metal dies evaluating marginal discrepancy.

h. In vitro trials utilizing extracted human teeth evaluating marginal discrepancy.

i. Studies reporting marginal discrepancy following luting.

j. Literature reviews.

k. Articles discussing slip casting and glass infiltration.

\section{Exclusion Criteria}

Articles and manuscripts discussing the following parameters were excluded:

a. Finite element analysis studies.

b. Case reports and case series.

c. Porcelain labial margins.

d. Marginal discrepancy in porcelain fused metal restorations.

e. Marginal discrepancy with partial veneer retainers.

f. Marginal discrepancy with labial veneers.

g. Porcelain repair systems creating marginal discrepancy.

h. Gingival porcelains.

\section{Results}

The database search yielded 70 articles out of which 47 articles were discarded after reading the abstract. Full texts were obtained for the remaining 25 articles. 16 articles were selected based on the inclusion criteria and 9 articles were excluded. The finally selected 16 articles were subjected to data extraction.

\section{Data Extraction}

The data from the finally included studied were tabulated and the following information were extracted.

1. Study design applied

2. CEBM levels of evidence.

3. Horizontal marginal discrepancy

4. Vertical marginal discrepancy.

5. Absolute marginal discrepancy.

6. Types of finish lines used.

7. Thickness of the die spacer.

8. Composition of ceramic processing materials.

9. Core and veneer mismatch.

10. Type of luting cements.

11. Method of measurement.

12. Instruments for measurement.

13. Statistical tests.

14. Processing techniques used. 


\section{Flow Chart For Search Stratergy:-}

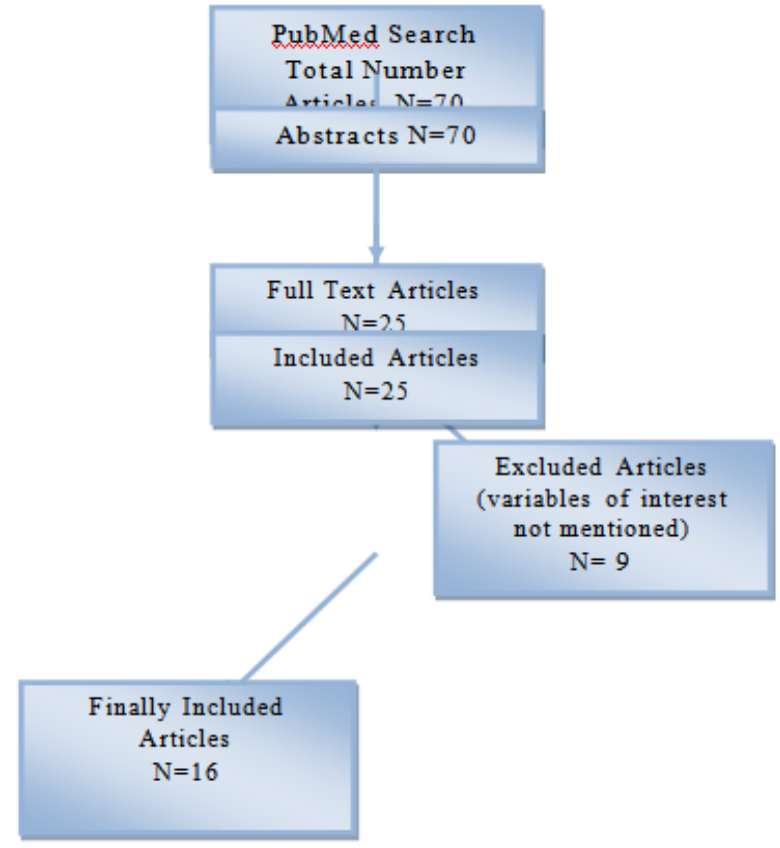

XII. Results:

Table 1 shows the variables of interest in the selected articles. The following information were extracted and tabulated.

Name of the author, study design, technique used for all ceramic fabrication, method and gadget for measuring marginal discrepancy, horizontal marginal discrepancy, vertical marginal discrepancy, absolute marginal discrepancy, type of luting cement, type of finish line, thickness of die spacer, comparision ceramic processing material, core and veneer mismatch, type of luting cements, instrument of measurement, statistical tests, processing technique were extracted and tabulated.

Table 2 shows the processed data for marginal discrepancy with conventional techniques (slip castimg,heat press and copy milling)

Graph 1 shows the forrest plot for conventional techniques(slip casting,heat pressing and copy milling)

Graph 2 shows the processed data for marginal discrepancy for conventional techniques

Table 3 shows the results of meta analysis for conventional techniques

Table 4 shows the processed data for marginal discrepancy with CAD/CAM techniques.

Table 5 shows the results for meta analysis of CAD/CAM techniques.

Graph 3 shows the processed data for marginal discrepancy with CAD/CAM techniques.

Graph 4 shows the forrest plot for marginal discrepancy with CAD/CAM techniques..

Table 6 shows the processed data for marginal discrepancy with conventional and CAD/CAM techniques.

Table 7 shows the processed data for marginal discrepancy with CAD/CAM technique and conventional techniques.

Graph 5 shows the processed data for marginal discrepancy with conventional and CAD/CAM techniques. techniques..

Graph 6 shows the forrest plot for marginal discrepancy with conventional and CAD/CAM

The results of the present systematic review has analysed the marginal discrepancy in all ceramic complete veneer retainers into three catergories.

\subsection{Catergory (1)}

Marginal discrepancy present in copy milling,Slip casting, and heat pressed ceramics techniques were included.Nine studies were included in catergory one and the statistics present in 
the respective articles were extracted and subjected to meta analysis and forrest plot was constructed subsequently. The results of the meta analysis stated the effect size or mean marginal discrepancy present in the three techniques grouped as

Others was estimated to be 56.53 microns(44.70-68.38) at 95 percent confidence interval.

\subsection{Catergory (2)}

Marginal discrepancy present in cad/cam ceramics included seven studies in catergory two and the statistics present in the respective studies extracted and subjected to meta analysis and forrestl plot was constructed subsequently. The results of the meta analysis stated the effect size or mean marginal discrepancy present in cad/cam technique was estimated to be 55.32 microns.(45.42 $65.27)$ at 95 percent confidence interval.

\subsection{Catergory (3)}

Three articles compared both the cad/cam and other technique for marginal discrepancy and the studies were grouped as catergory 3 and a higher reliability could be attributed since both the technique were compared using the same methodology. The results of meta analysis calculated the overall estimate for marginal discrepancy for all ceramic complete veneer restorations as 43.8(29.3$25.83)$ microns at 95 percent confidence interval $(\mathrm{p}<.05)$.

TABLE:1-VARIABLES OF INTEREST IN SELECTED ARTICLES

\begin{tabular}{|c|c|c|c|c|c|c|c|c|c|c|c|c|c|c|}
\hline $\begin{array}{c}\text { Name of the } \\
\text { author }\end{array}$ & Study Design & Technique Used & $\begin{array}{l}\text { Method of } \\
\text { Measurement }\end{array}$ & \begin{tabular}{|c} 
Horizontal \\
Marginal \\
Disctepancy
\end{tabular} & $\begin{array}{c}\text { Nertical } \\
\text { Marginal } \\
\text { Discrepancy }\end{array}$ & $\begin{array}{l}\text { Absolute } \\
\text { Marginal } \\
\text { Discrepancy }\end{array}$ & $\begin{array}{c}\text { Type of } \\
\text { Finish Line } \\
\text { Used }\end{array}$ & $\begin{array}{l}\text { Thickness of } \\
\text { Dyes spacer }\end{array}$ & $\begin{array}{c}\text { Comparison of } \\
\text { Ceramic } \\
\text { processing } \\
\text { material }\end{array}$ & $\begin{array}{l}\text { Core and } \\
\text { veneer } \\
\text { Mismatch }\end{array}$ & $\begin{array}{l}\text { Type of } \\
\text { luting } \\
\text { Cements }\end{array}$ & $\begin{array}{l}\text { Instrument for } \\
\text { Measurement }\end{array}$ & Stats test & $\begin{array}{l}\text { Processing } \\
\text { Technique }\end{array}$ \\
\hline \begin{tabular}{|c|} 
Sulaiman et \\
al 1997
\end{tabular} & $\begin{array}{c}\text { Experimental PS } \\
\text { Study Invitro }\end{array}$ & $\begin{array}{l}\text { Slip casting and } \\
\text { heat press } \\
\text { Teechnique }\end{array}$ & $\begin{array}{c}\text { Digital } \\
\text { Microssoope }\end{array}$ & Not Mentioned & Not Mentioned & Mentioned & $\begin{array}{c}\text { Not } \\
\text { mentioned }\end{array}$ & Mentioned & Mentioned & Mentioned & $\begin{array}{c}\text { Not } \\
\text { Mentioned }\end{array}$ & Mictoscope & $\begin{array}{l}3 \text { way anova Tukey } \\
\text { Post Hoc Analysis }\end{array}$ & $\begin{array}{l}\text { Slip casting and } \\
\text { heat press } \\
\text { technique }\end{array}$ \\
\hline $\begin{array}{c}\text { Matity ct al } \\
1989\end{array}$ & $\begin{array}{l}\text { Experimental } \\
\text { P.S. Invitro }\end{array}$ & Conventional & \begin{tabular}{|c|} 
Video Enhanced \\
Microscope
\end{tabular} & Not Mentioned & Not Mentioned & entioned & entioned & Mentioned & Mentioned & $\begin{array}{c}\text { Not } \\
\text { Mentioned }\end{array}$ & Mentioned & \begin{tabular}{|c|} 
Video Enhanced \\
Mictoscope
\end{tabular} & Not Mentioned & Conventional \\
\hline $\begin{array}{c}\begin{array}{c}\text { Rinke et al } \\
1995\end{array} \\
\end{array}$ & $\begin{array}{l}\text { Experimental } \\
\text { P.S Invitro }\end{array}$ & Slip casting & Light Microssope & Not Mentioned & Not Mentioned & Mentioned & entioned & $\begin{array}{c}\text { Not } \\
\text { Mentioned }\end{array}$ & Mentioned & $\begin{array}{c}\text { Not } \\
\text { Mentioned }\end{array}$ & $\begin{array}{c}\text { Not } \\
\text { Mentioned }\end{array}$ & $\begin{array}{c}\text { Light } \\
\text { Microscope }\end{array}$ & Kruskal Wallis Test & Slip Casting \\
\hline \begin{tabular}{|c|} 
Rinke et al \\
2012
\end{tabular} & $\begin{array}{c}\text { Experimental } \\
\text { P.S Invitro }\end{array}$ & Cad cam & \begin{tabular}{|c|} 
Light Microscope \\
and processing \\
Software
\end{tabular} & Mentioned & Not Mentioned & Mentioned & $\begin{array}{l}\text { Not } \\
\text { mentioned }\end{array}$ & Mentioned & Mentioned & $\begin{array}{c}\text { Not } \\
\text { Mentioned }\end{array}$ & $\begin{array}{c}\text { Not } \\
\text { Mentioned }\end{array}$ & $\begin{array}{c}\text { Light } \\
\text { Microscope }\end{array}$ & 2 way anova & cad/cam \\
\hline \begin{tabular}{|c|} 
Bindi et al \\
2005
\end{tabular} & $\begin{array}{l}\text { Experimental } \\
\text { P.S. Invitro }\end{array}$ & \begin{tabular}{|c|} 
Slip cast , heat \\
pressing and \\
cadcam
\end{tabular} & Light Microscope & Not Mentioned & Not Mentioned & Mentioned & entioned & $\begin{array}{c}\text { Not } \\
\text { Mentioned }\end{array}$ & Mentioned & $\begin{array}{c}\text { Not } \\
\text { Mentioned }\end{array}$ & $\begin{array}{c}\text { Not } \\
\text { Mentioned }\end{array}$ & $\begin{array}{c}\text { Light } \\
\text { Microscope }\end{array}$ & Scheffe test & $\begin{array}{c}\text { Slip Cast,heat } \\
\text { pressing and cad } \\
\text { cam }\end{array}$ \\
\hline $\begin{array}{c}\text { Farid et al } \\
2012\end{array}$ & $\begin{array}{c}\text { Experimental } \\
\text { P.S.Invitro }\end{array}$ & Conventional & Stereo Miroscope & Not Mentioned & Not Mentioned & Mentioned & $\begin{array}{l}\text { Not } \\
\text { mentioned }\end{array}$ & $\begin{array}{c}\text { Not } \\
\text { Mentioned }\end{array}$ & Mentioned & Mentioned & $\begin{array}{l}\text { Not } \\
\text { Ientioned }\end{array}$ & $\begin{array}{c}\text { Sterio } \\
\text { Microscope }\end{array}$ & Not Mentioned & Conventional \\
\hline $\begin{array}{c}\text { Beschnidt et } \\
\text { al } 1999\end{array}$ & \begin{tabular}{||c|c|} 
Experimental \\
P.S.Invitro
\end{tabular} & $\begin{array}{l}\text { Staining } \\
\text { Veneering } \\
\text { Technique }\end{array}$ & $\begin{array}{c}\text { Stcrece } \\
\text { Mirascope,and } \\
\text { Analysis 2.1 }\end{array}$ & Not Mentioned & Not Mentioned & Mentioned & entioned & $\begin{array}{c}\text { Not } \\
\text { Mentioned }\end{array}$ & Mentioned & $\begin{array}{c}\text { Not } \\
\text { Mentioned }\end{array}$ & Mentioned & \begin{tabular}{|c|} 
Stcreo \\
Microscope,and \\
Analysis 2.1 \\
\end{tabular} & $\begin{array}{l}\text { Kruskal Wallis } \\
\text { Test, Will } \\
\text { Willcoxin }\end{array}$ & $\begin{array}{l}\text { Straining } \\
\text { Veneering } \\
\text { Technique }\end{array}$ \\
\hline $\begin{array}{c}\text { Okutan et al } \\
2006\end{array}$ & \begin{tabular}{|c|} 
Experimental \\
P.S. Invitro
\end{tabular} & Cad cam & $\begin{array}{c}\text { Stereo } \\
\text { Mictoscope }\end{array}$ & Not Mentioned & Not Mentioned & Mentioned & entioned & $\begin{array}{c}\text { Not } \\
\text { Mentioned }\end{array}$ & Mentioned & $\begin{array}{c}\text { Not } \\
\text { Mentioned }\end{array}$ & Mentioned & Mictroscope & \begin{tabular}{|c|} 
Paied T-Test, Will \\
coxil
\end{tabular} & Cad cam \\
\hline \begin{tabular}{|c|}
$\begin{array}{c}\text { Suarez et al } \\
2003\end{array}$ \\
\end{tabular} & \begin{tabular}{|c|} 
Experimental \\
P.S. Invitro
\end{tabular} & Cad cam & \begin{tabular}{|c|} 
Image Analysis \\
Program
\end{tabular} & Mentioned & Mentioned & Mentioned & entioned & $\begin{array}{c}\text { Not } \\
\text { Mentioned }\end{array}$ & Mentioned & Mentioned & $\begin{array}{c}\text { Not } \\
\text { Mentioned }\end{array}$ & \begin{tabular}{|c|} 
image analysis \\
program
\end{tabular} & \begin{tabular}{|c|} 
Student's T Test \\
and Pairesd T Test
\end{tabular} & Cad cam \\
\hline $\begin{array}{c}\text { Korkut et al } \\
2011\end{array}$ & $\begin{array}{c}\text { Experimental } \\
\text { P.S.Invitro }\end{array}$ & $\begin{array}{l}\text { Cad cam and } \\
\text { heat Press }\end{array}$ & $\begin{array}{c}\text { Lucia G Vetsion } \\
4.51\end{array}$ & Not Mentioned & Not Mentioned & Mentioned & entioned & $\begin{array}{c}\text { Not } \\
\text { Mentioned }\end{array}$ & Mentioned & $\begin{array}{c}\text { Not } \\
\text { Mentioned }\end{array}$ & Mentioned & $\begin{array}{c}\text { Lucia G Version } \\
4.51\end{array}$ & \begin{tabular}{|c|} 
Welch Test,Dunnet \\
C test, Kruskal test \\
Chi square test
\end{tabular} & $\begin{array}{l}\text { Cad cam and } \\
\text { Heal Press }\end{array}$ \\
\hline $\begin{array}{c}\text { Quintas et al } \\
2004\end{array}$ & \begin{tabular}{||c|c|} 
Experimental \\
P.S.Invitra
\end{tabular} & \begin{tabular}{|c|}
$\begin{array}{c}\text { Cad cam,slip } \\
\text { cast and heat } \\
\text { Pressing }\end{array}$ \\
\end{tabular} & Profile Projector & Not Mentianed & Mentioned & Mentioned & entioned & $\begin{array}{c}\text { Not } \\
\text { Mentioned }\end{array}$ & Mentioned & $\begin{array}{c}\text { Not } \\
\text { Mentioned }\end{array}$ & Mentioned & Profile Projector & 4 way Anowa & \begin{tabular}{|c|} 
Cad cam, slip \\
casting, Heat \\
Pressing
\end{tabular} \\
\hline $\begin{array}{c}\text { Cardelli ict } \\
\text { al } 2011\end{array}$ & $\begin{array}{l}\text { Expcrimental } \\
\text { P.S. In vivo }\end{array}$ & \begin{tabular}{|c|} 
Chair side \\
Milling and Cad \\
Cam
\end{tabular} & Microscope & Nat Mentioned & Not Mentioned & Mentioned & $\begin{array}{c}\text { Not } \\
\text { mentioned }\end{array}$ & $\begin{array}{c}\text { Not } \\
\text { Mentioned }\end{array}$ & Mentioned & $\begin{array}{c}\text { Not } \\
\text { Mentioned }\end{array}$ & Mentioned & Mictoscope & \begin{tabular}{|l|} 
Anderson darling \\
Test and Mann - \\
Whitney U Test
\end{tabular} & $\begin{array}{c}\text { Chair Side } \\
\text { Milling and Cad } \\
\text { cam }\end{array}$ \\
\hline \begin{tabular}{|c|} 
Schaefr et \\
al2012
\end{tabular} & $\begin{array}{l}\text { Experimental } \\
\text { P.S. Invitro }\end{array}$ & $\begin{array}{l}\text { Heat Press } \\
\text { Ceramic }\end{array}$ & \begin{tabular}{|c|}
$\begin{array}{c}\text { Structured Light } \\
\text { scanner }\end{array}$ \\
\end{tabular} & Not Mentioned & Not Mentioned & Mentioned & Mentioned & $\begin{array}{c}\text { Not } \\
\text { Mentioned }\end{array}$ & $\begin{array}{l}\text { Ceramic } \\
\text { System }\end{array}$ & $\begin{array}{c}\text { Not } \\
\text { Mentioned }\end{array}$ & $\begin{array}{c}\text { Not } \\
\text { Mentioned }\end{array}$ & $\begin{array}{c}\text { Structured Light } \\
\text { Scanner }\end{array}$ & Levene's Test & $\begin{array}{l}\text { Heat Press } \\
\text { Ceramic }\end{array}$ \\
\hline $\begin{array}{c}\text { Nakamura ot } \\
\text { al } 2000\end{array}$ & $\begin{array}{l}\text { Experimental } \\
\text { P.S. Invitro }\end{array}$ & $\begin{array}{c}\text { Copy Milling } \\
\text { System and } \\
\text { Conventional } \\
\text { System }\end{array}$ & Profile Projector & Not Mentioned & Not Mentioned & Mentioned & Mentioned & Mentioned & Mentioned & $\begin{array}{c}\text { Not } \\
\text { Mentioned }\end{array}$ & $\begin{array}{c}\text { Not } \\
\text { Mentioned }\end{array}$ & Profile Projector & $\begin{array}{l}\text { Bond and fertroni } \\
\text { Test, Student T test }\end{array}$ & $\begin{array}{c}\text { Copy Milling } \\
\text { System and } \\
\text { Conventional } \\
\text { System }\end{array}$ \\
\hline $\begin{array}{c}\text { Kohorst et } \\
\text { al } 2009\end{array}$ & $\begin{array}{l}\text { Experimental } \\
\text { P. S.Invitra }\end{array}$ & Cad cam & $\begin{array}{l}\text { Light Optical } \\
\text { Microscope }\end{array}$ & Mentioned & Mentioned & Mentioned & $\begin{array}{c}\text { Not } \\
\text { mentioned }\end{array}$ & $\begin{array}{c}\text { Not } \\
\text { Mentioned }\end{array}$ & Mentioned & $\begin{array}{c}\text { Not } \\
\text { Mentioned }\end{array}$ & Mentioned & $\begin{array}{l}\text { Light Optical } \\
\text { Microscope }\end{array}$ & \begin{tabular}{|c|} 
I way \\
Anowa, Kol mogorov- \\
Smirnor and \\
Levene, Scheffe \\
Test
\end{tabular} & Cad cam test \\
\hline $\begin{array}{l}\text { Lee et al } \\
2007\end{array}$ & $\begin{array}{c}\text { Experimental } \\
\text { P.S. Invitra }\end{array}$ & Cad cam & Microscope & t Mentioned & Not Mentioned & Mentioned & $\begin{array}{c}\text { Not } \\
\text { mentioned }\end{array}$ & $\begin{array}{c}\text { Not } \\
\text { Mentioned }\end{array}$ & Ment & $\begin{array}{c}\text { Not } \\
\text { Mentioned }\end{array}$ & $\begin{array}{c}\text { Not } \\
\text { Mentioned }\end{array}$ & Microscope & $\begin{array}{c}\text { Tukey's Test and I } \\
\text { way anova }\end{array}$ & Cad cam \\
\hline
\end{tabular}

TABLE: 2- PROCESSED DATA FOR MARGINAL DISCREPANCY WITH CONVENTIONAL TECHNIQUES(SLIP CASTING, HEAT PRESS AND COPY MILLING)

\begin{tabular}{|l|l|l|l|l|}
\hline AUTHOR & YEAR & No studied & Mean $\mu \mathrm{m}$ & SE \\
\hline ABBATE ET AL & 1989 & 80 & 54.7 & 1.677517 \\
\hline RINKE ET AL & 1995 & 160 & 40.125 & 2.015952 \\
\hline SULAIMAN ET AL & 1997 & 90 & 102.1033 & 4.399093 \\
\hline BESCHNIDT ET AL & 1999 & 60 & 72.4 & 2.891828 \\
\hline NAKAMURA ET AL & 2000 & 450 & 58.6 & 0.812483 \\
\hline
\end{tabular}




\begin{tabular}{|l|l|l|l|l|} 
QUINTAS ET AL & 2004 & 30 & 50 & 6.441187 \\
\hline KORKUT ET AL & 2011 & 90 & 46.94 & 0.87458 \\
\hline FARID ET AL & 2012 & 120 & 29.93333 & 0.183548 \\
\hline SCHAEFER ET AL & 2012 & 10 & 56 & 5.09902 \\
\hline
\end{tabular}

TABLE: 3-RESULT OF META ANALYSIS: CONVENTIONAL TECHNIQUES (SLIP CASTING, HEAT PRESS AND COPY MILLING)

\begin{tabular}{|c|c|c|c|}
\hline Study & $\begin{array}{l}\text { Effect } \\
\text { size } \mu \mathrm{m}\end{array}$ & \multicolumn{2}{|c|}{$\begin{array}{cc} & {[95 \% \mathrm{Cl}]} \\
\text { Lower limit } & \text { Upper limit } \\
\mu \mathrm{m} & \end{array}$} \\
\hline ABBATE ET AL(1989) & 54.7 & 51.412 & 57.988 \\
\hline RINKE ET AL (1995) & 40.125 & 36.174 & 44.076 \\
\hline SULAIMAN ET AL(1997) & 102.103 & 93.481 & 110.725 \\
\hline BESCHNIDT ET AL (1999) & 72.4 & 66.732 & 78.068 \\
\hline NAKAMURA ET AL (2000) & 58.6 & 57.008 & 60.192 \\
\hline QUINTAS ET AL (2004) & 50 & 37.376 & 62.624 \\
\hline KORKUT ET AL (2011) & 46.94 & 45.226 & 48.654 \\
\hline FARID ET AL (2012) & 29.933 & 29.574 & 30.293 \\
\hline SCHAEFER ET AL(2012) & 56 & 46.006 & 65.994 \\
\hline Pooled ES & 56.535 & 44.706 & 68.364 \\
\hline
\end{tabular}

ES - Effect size (mean)

Heterogeneity chi-squared $=2158.77$ (d.f. $=8) \mathrm{p}=0.000$

Variation in ES attributable to heterogeneity $=99.6 \%$

The overall estimate is 56.5 with (95\% C I 44.7-68.4)

\section{GRAPH: 1}

FORREST PLOT FOR CONVENTIONAL TECHNIQUES (SLIP CASTING, HEAT PRESS AND COPY MILLING)

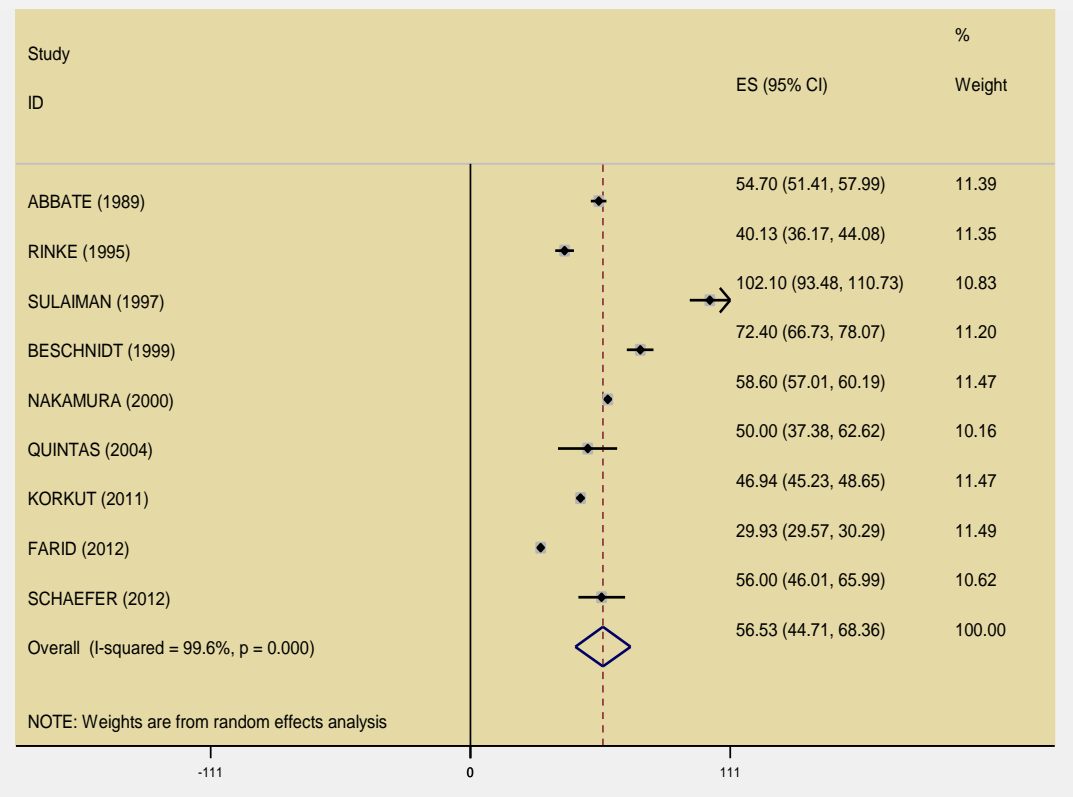


GRAPH: 2

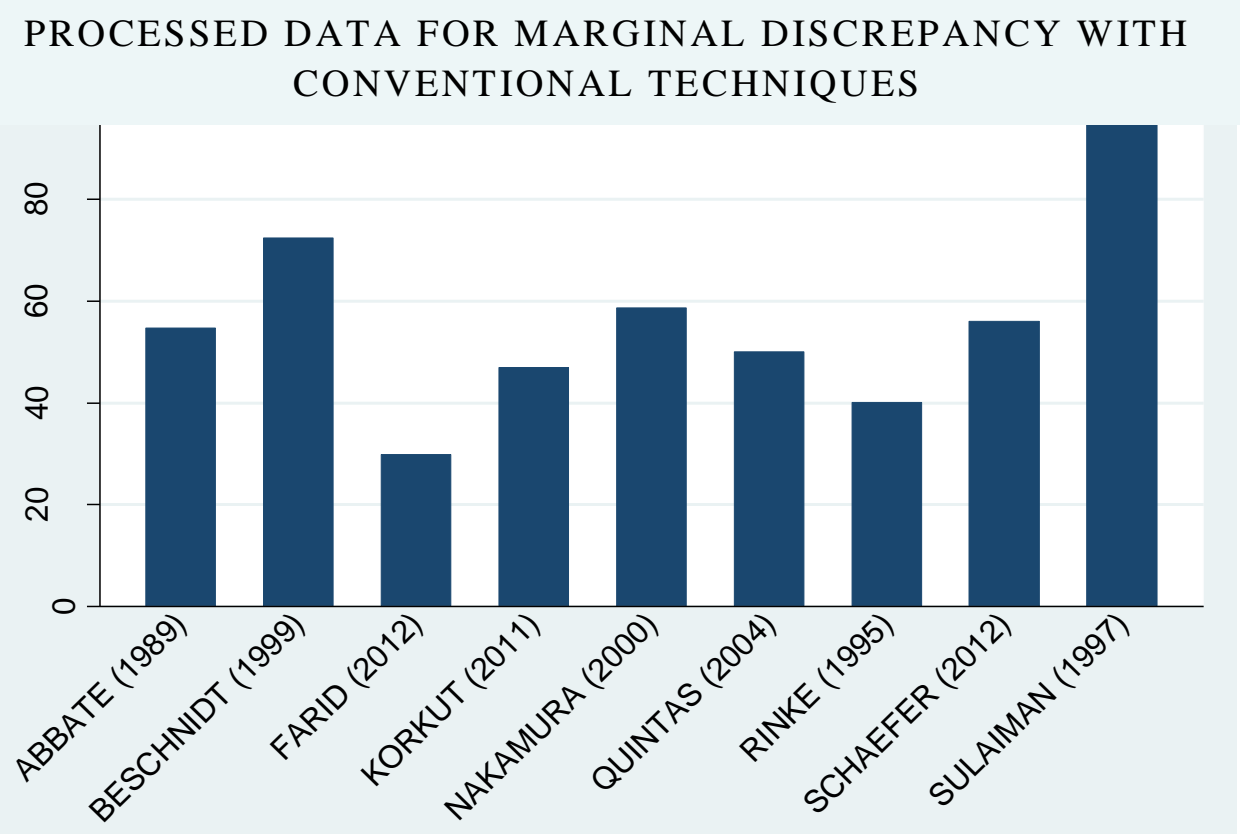

TABLE: 4-PROCESSED DATA FOR MARGINAL DISCREPANCY WITH CAD/CAM TECHNIQUE

\begin{tabular}{|l|l|l|l|l|}
\hline Study & Year & No studied & MEAN $\mu \mathrm{m}$ & SE \\
\hline SUAREZ ET AL & 2003 & 40 & 107 & 45.63442 \\
\hline BINDL ET AL & 2005 & 24 & 27.25 & 18.69492 \\
\hline OKUTAN ET AL & 2006 & 64 & 32.85 & 6.750185 \\
\hline LEE ET AL & 2008 & 60 & 85.56667 & 9.553533 \\
\hline KOHORST ET AL & 2009 & 160 & 159.05 & 34.54457 \\
\hline CARDELLI ET AL & 2011 & 148 & 113.935 & 60.65711 \\
\hline RINKE ET AL & 2012 & 180 & 25.9567 & 24.71504 \\
\hline
\end{tabular}

TABLE: 5 RESULTS OF META ANALYSIS:CAD/CAM TECHNIQUE

\begin{tabular}{|l|c|c|c|}
\hline \multicolumn{1}{|c|}{ Study } & & \multicolumn{2}{c|}{$\begin{array}{c}{[95 \% \text { C I] }} \\
\text { Upper limit } \\
\mu \mathrm{m}\end{array}$} \\
\hline & Effect size $\mu \mathrm{m}$ & \multicolumn{2}{|c|}{} \\
\hline & & 17.558 & 196.442 \\
\hline SUAREZ ET AL(2003) & 107 & -9.391 & 63.891 \\
\hline BINDL ET AL (2005) & 27.25 & 19.62 & 46.08 \\
\hline LEUTAN ET AL (2006) & 32.85 & 66.842 & 104.291 \\
\hline KOHORST ET AL (2009) & 85.567 & 91.344 & 226.756 \\
\hline CARDELLI ET AL(2011) & 159.05 & -4.951 & 232.821 \\
\hline RINKE ET AL(2012) & 113.935 & 77.516 & 174.397 \\
\hline & 125.957 & 45.426 & 65.278 \\
\hline Pooled Effect size & 55.352 & & \\
\hline
\end{tabular}

Heterogeneity chi-squared $=42.76($ d.f. $=6) \mathrm{p}=0.000$

Variation in ES attributable to heterogeneity $=86.0 \%$ Pooled estimate is 55.352 (95\% C I 45.4-65.3) 
GRAPH: 3

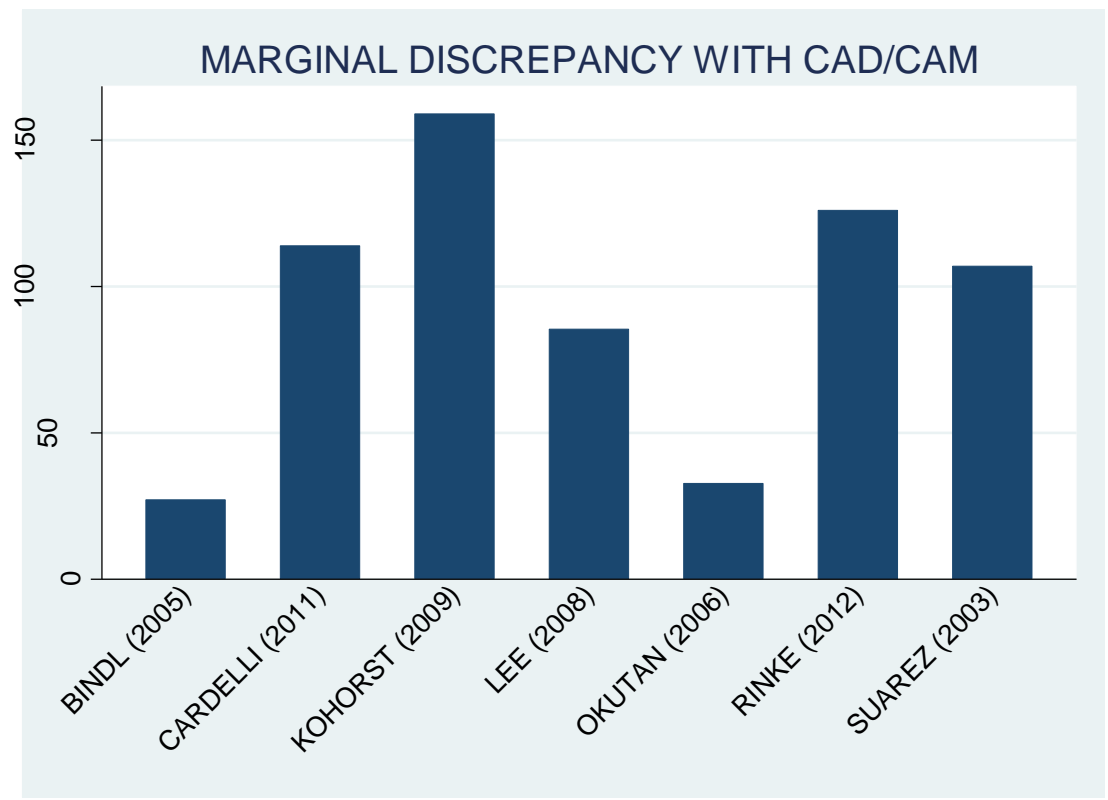

GRAPH: 4

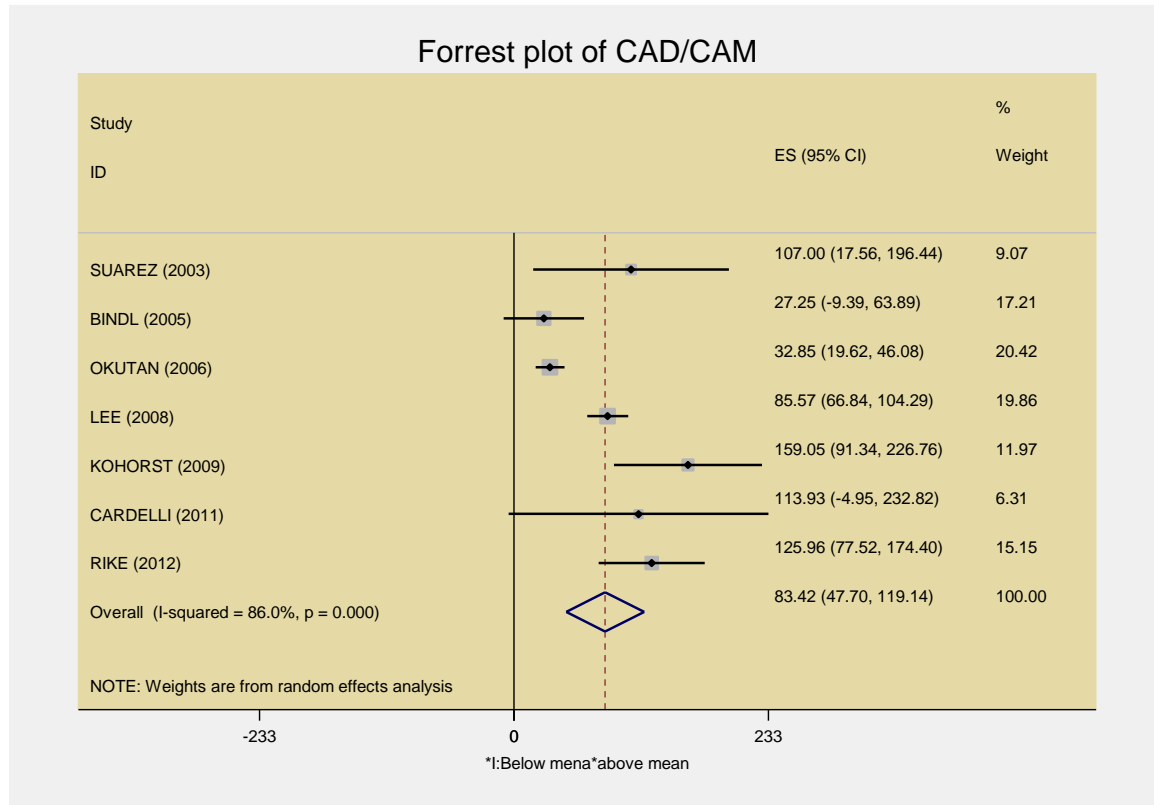

TABLE: 6-PROCESSED DATA FOR MARGINAL DISCREPANCY WITH CAD/CAM AND CONVENTIONALTECHNIQUES

\begin{tabular}{|c|c|c|c|c|}
\hline Study & Year & Mean $\mu \mathrm{m}$ & SE & No. Studied \\
\hline KORKUT ET AL & 2011 & 46.94 & 8.296991 & 90 \\
\hline BINDAL ET AL & 2005 & 27.25 & 18.24437 & 24 \\
\hline QUINTAS ET AL & 2004 & 50 & 35.27983 & 30 \\
\hline
\end{tabular}

TABLE: 7-RESULTS OF META ANALYSIS: CAD/CAM AND CONVENTIONAL TECHNIQUES

\begin{tabular}{|l|c|c|c|c|}
\hline Study & Effect $\mu \mathrm{m}$ & [95\% Conf. Interval] & Up Weight \\
\hline & & Lower limit & Upper limit & 63.202 \\
\hline KOR KUT ET AL (2011) & 46.940 & 30.678 & 79.23 \\
\hline BINDAL ET AL (2005) & 27.250 & -8.508 & 63.008 & 16.39 \\
\hline QUINTAS ET AL (2004) & 50.000 & -19.147 & 119.147 & 4.38 \\
\hline Pooled Effect & 43.848 & 29.373 & 58.323 & 100.00 \\
\hline
\end{tabular}


Chi-squared (variation in ES attributable to heterogeneity) $=0.0 \%$

Heterogeneity was not absorbed

The overall estimate was $43.8 \%$ (C I $95 \% 29.3$ to 58.3 )

\section{GRAPH: 5}

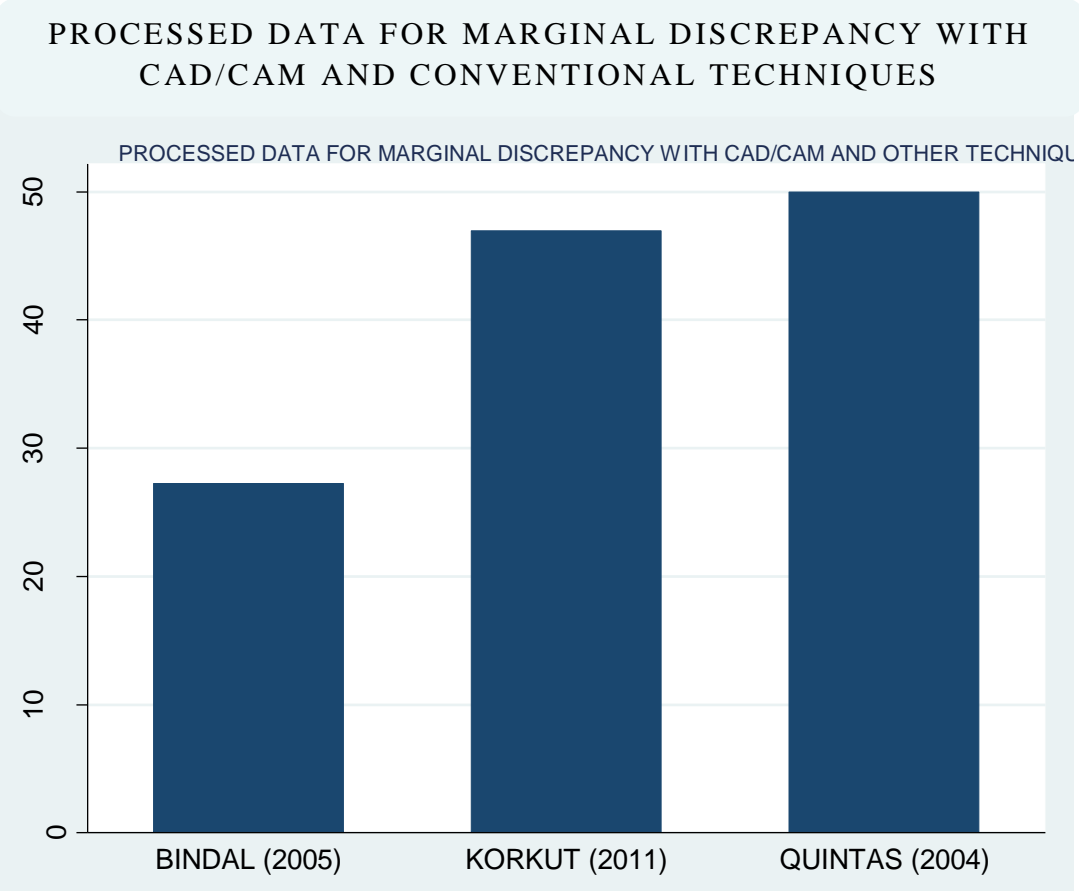

GRAPH: 6

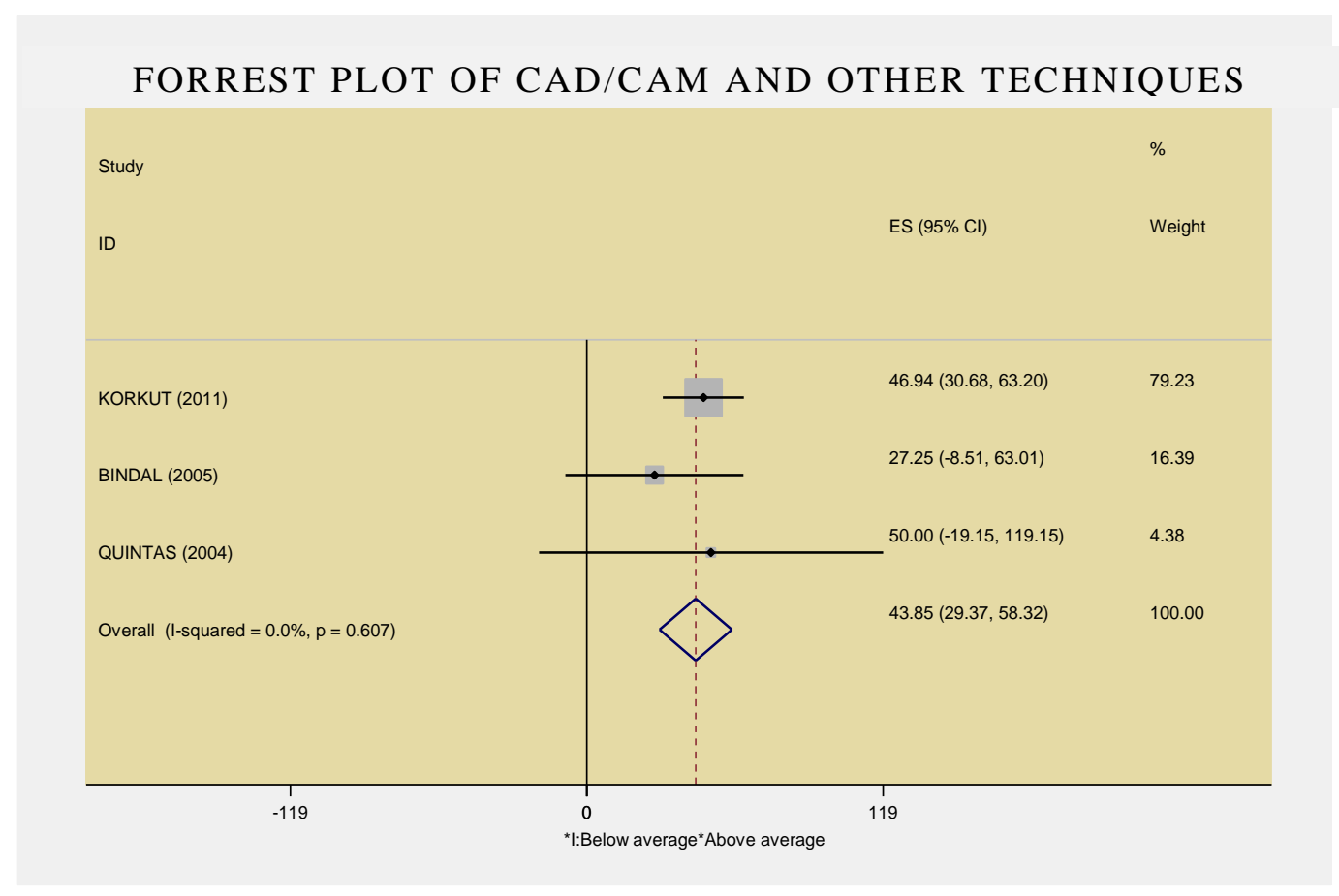

XIII. Discussion:

All ceramic crowns are the most biocompatible and most aesthetic of all the biomaterials used in restoring the teeth , The construction of all ceramic crowns involves the fabrication of high strength ceramic coping which offers remarkable resistance against occlusal and functional loading 
.Apart from fracture resistance and aesthetics, the marginal accuracy of the crowns is valued as one of the most important criteria for clinical performance and success in clinical situations.Increased marginal discrepancy of a crown acclerates the rate of luting cement dissolution ,and increases the chances for microleakage.This microleakage can invoke inflammatory response in a vital pulp tissue. Compromised marginal accuracy increases the probability of plaque retention and changes the composition of subgingival microflora precipitating the onset and progression of periodontal disease.

The ceramic crown coping can be constructed from various high strength ceramic biomaterials by various manufacturing processes. The slip casting technique has been well estabilished for the fabrication of coping as in Inceram glass infiltration ceramic systems.. Modifications of the chemical and structural composition of the inceram infiltration ceramic result in increased strength in the ceramic core, over which layering of veneering ceramic is done. To fabricate a slip cast crown , a refractory die is needed to compensate for the shrinkage of the slip during the sintering process. The slip is loaded on the plaster models using ceramic brush form the crown coping. Then the material is sintered to a open porous state and infiltrated with lanthnum glass to provide the final strength.

The heat pressing technique can be used for the fabrication of copings as in Empress 2 lithium disilicate ceramics. The heat pressing technique is based on the lost wax principle. Prefabricated ceramic ingots of lithium disilicate ceramic are heated at high temperatures and then pressed into the lost wax form of crown coping. The coping is finished, over which layering of veneering ceramic is done for the final prosthesis.

Copy milling is another technique employed in all ceramic fabrication .Here a resin wax pattern is fabricated over the refractory die and laser scan is used which subsequently transfers the scanned image and a ceramic core is fabricated

$\mathrm{CAD} / \mathrm{CAM}$ ( computer aided designing and computer aided milling) processing technique uses scanning design and milling process to customize and shape the coping from industrially pre fabricated ceramic blocks. The CAD/CAM equipment can be used in house, such as cerec inlab and decim system comprising the scanning device ,the design unit and measuring unit.The CAD/CAM technology is also used in measuring centres such as procera and decim system. The typical work organization of machine centre requires that the restoration data must be acquired in the local dental lab.The acquired data are then sent via modem and the coping will be machined and will be sent back physically to the dental lab where the all ceramic core crowns will be finalised and delivered to the dentist.Recently cerec3 system have been launched which utilizes a direct intra oral scanning method to capture the images and the captured images transferred to the computer where the restoration is designed and the data is directly sent to the milling machine where the crown is fabricated.

This CADCAM technique offers a great advantage over conventional ceramic processing techniques by eliminating the clinical steps in impression making and laboratory steps including cast pouring,articulation, die sectioning, casting and subsequent layering, thus saving enormous amount of time and manpower.However the results of this systematic review negated the null hypothesis and inferred there is no difference in the amount of marginal discrepancy with both the conventional and CADCAM processing techniques.

Abbate et al(1989) proposed a studied the marginal fit of1)Metal ceramic crowns with a metal-butt facial margin.2)Metal ceramic crowns with a porcelain -butt facial margin3)Cerestore crowns4)Dicor crowns and reported significant differences in marginal fit in the all ceramic crowns. Rinke et al (1995) studied the marginal adaptation and fracture resistance of copy-milled and conventional In-Ceram crowns. The marginal accuracy of the copy-milled units ranged from 6 to 153 micrometer, and that of the conventional fabricated units ranged from 1 to 153 micrometer.

Sulaiman et al(1997 studied the marginal discrepancies of all -ceramic crown systems at various surfaces of the restoration:mesial,distal,buccal,and lingual.The lingual surfaces of the ceramic crowns possessed significantly greater marginal discrepancies than all other surfaces. There were no significant differences among the other three surfaces:facial,mesial and distal.

Beschindt et al(1999) compared the marginal fit of five different all ceramic crown systems In Ceram,Empress staining technique.Empress veneering technique,Celay feldspathic system,CeLAY In Ceram.The marginal discrepancies found in this study were all within biologically acceptable standards. Suarez et al(2003) studied the influence of two finish line configurations on the marginal accuracy of Procera AllCeram crowns. The marinal gap was within the range of clinical acceptability. 
Lee et al(2008) studied the accuracy of marginal and internal fit between the all-ceramic crowns manufactures by a conventional double layer computer aided design/computer aided manufacturing(CAD/CAM $)$ system and a single layer system. There were no significant differences in marginal discrepancy between the doble-layer type CAD/CAM system(Procera) and single-layer type CAD/CAM(Cerec3D).

Kohorst et al(2009) evaluated the marginal fit of four -unit zirconia bridge frameworks fabricated using four different computer -aided design(CAD)/computer- aided manufacturing(CAM)systems and concluded four-unit FPDs made from fully sintered zirconia show better marginal accuracy than restorations made from presintered material.

Cardelli et al(2011) conducted a clinical trial to test the accuracy of single all- ceramic zirconia crowns resulting from digital intraoral impressions with active wavefront sampling technique by measuring the marginal and internal fit of the crowns.Single crown restorations obtained using digital intraoral impressions based on active wavefront sampling technology presented enough accuracy to be used as an alternative to the conventional impression technique.

Farid et al(2012) evaluated the influence of core thickness and fabrication stages on the marginal accuracy of IPS e.max Press crowns. The IPSe.max Press crowns have an acceptable marginal fit.

Rinke et al(2012) evaluated the effect of different parameters on the marginal discrepancy of CAD/CAM- fabricated with different scanning and milling procedures, and concluded manual adjustment seems to be necessary for all systems tested to achieve maximum marginal gaps below the threshold of 120 micrometer.

Bindl et al (2005) evaluted and concluded the marginal and internal fit of all -ceramic molar copings hypothesizing that computer aided design/computer aided manufacturing (CAD/CAM) fabrication shows the same accuracy of fit as conventional techniques.

Quintas et al((2004) studied the effect of different finish lines, ceramic manufacturing techniques and luting agents on the vertical discrepancy of ceramic copings.The ceramic manufacturing technique was the only significant factor tested that influenced vertical marginal discrepancy when the factor was considered separately after cementation procera alumina copings presented the lowest discrepancy for all experimental conditions.

Korkut et al (2011) compared the marginal and internal fit and also microleakage of zirconia infrastructures (Procera, All-Zircon. Cercon Smart Ceramics) in contrast to heat -pressed ones (Empress2) and reported significant differences between them.

The other factors influencing marginal discrepancy include

Type of finish lines influencing marginal discrepancy, Thickness of the die spacer, Composition of ceramic processing materials, Firing sequence of ceramic materials, Core and veneer mismatch, Dissolution of luting cements,.Abrasion of margins with brushing technique respectively.The commonly used finish lines for all ceramic complete veneer retainers are rounded shoulder and heavy chamfer with a uniform reduction of $2 \mathrm{~mm}$ respectively. The axial taper of the tooth preparation vary between 6 to 20 degree and studies have revealed the best marginal fit of $\mathrm{cad} / \mathrm{cam}$ restoration is achieved with a larger convergence angle of 12 to 15 degree and shoulder preparation exhibited statistically higher discrepancy when compared to a heavy chamfer margin. As far as influence of core thickness and fabrication techniques the authors have observed a statistically significant increase in marginal discrepancy following veneering and layering .However after glazing no significant increase in gap were detected.However ,Farid and balkaya et al noted a significant difference but sulaiman et al found no significant difference in various stages of crown fabrication.

The luting cements play a significant role in marginal discrepancy. The luting agents had a significant effect on the marginal fit of zirconia based crowns. Resin composite cement resulted in larger marginal openings than glass ionomer cement.The cementation increases the marginal gap between the crown and the preparation for the ceramic systems regardless of the type of the cement evaluated.

Dissolution of luting cements is well documented in the literature and the primary etiology constituting this phenomenon include variation in salivary ph,quantity of gingival crevicular fluid,plaque accumulation,microbial colonisation which subsequently leads to dissolution of luting cements inducing microleakage and marginal discrepancy.Resin cements are the most resistant to dissolution followed by resin modified glass ionomer and conventional glass ionomer.

Abrasion of the ceramic margins due to long term effects of tooth brushing has not been well documented in the literature..In this systematic review even though the marginal discrepancy present in $\mathrm{cad} / \mathrm{cam}$ technique shows a statistically significant difference than the other techniques 
of fabrication but Clinical significance may not be present in routine clinical practice .Long term in vivo studies could be done to investigate this process through carefully formulated randomized controlled trials.

\section{Conclusion:}

Within the limitations of the present systematic review marginal discrepancy induced by $\mathrm{CAD} / \mathrm{CAM}$ technique 55.352(54.4-65.3)microns at 95 percent confidence interval and conventional techniques was estimated to be 56.53 microns $(44.70-68.38)$ at 95 percent confidence interval.The marginal discrepancy induced by cad/cam and other conventional techniques viz slip casting,copy milling, heat pressing are similar and both the systems can be employed to fabricate all ceramic complete veneer retainers.

\section{References:}

[1]. Rinke S, Fornefett D, Gersdorff N, Lange K, Roediger M .Multifactorial analysis of the impact of different manufacturing processes on the marginal fit of zirconia copings. Dent Mater J. 2012;31(4):601-9.

[2]. Schaefer O, Watts DC, Sigusch BW, Kuepper H, Guentsch A. Marginal and internal fit of pressed lithium disilicate partial crowns in vitro: a three-dimensional analysis of accuracy and reproducibility. Dent Mater. 2012 Mar;28(3):320-6. doi: 10.1016/j.dental.2011.12.008. Epub 2012 Jan 21.

[3]. Korkut L, Cotert HS, Kurtulmus H. Marginal, internal fit and microleakage of zirconia infrastructures: an in-vitro study. Oper Dent. 2011 Jan-Feb;36(1):72-9. doi: 10.2341/10-107-LR1. Epub 2011 Mar 24.

[4]. Kohorst P, Brinkmann H, Li J, Borchers L, Stiesch M. Marginal accuracy of four-unit zirconia fixed dental prostheses fabricated using different computer-aided design/computer-aided manufacturing systems. Eur J Oral Sci. 2009 Jun;117(3):319-25. doi: 10.1111/j.1600-0722.2009.00622.x.

[5]. Okutan M, Heydecke G, Butz F, Strub JR. Fracture load and marginal fit of shrinkage-free $\mathrm{ZrSiO} 4$ all-ceramic crowns after chewing simulation. J Oral Rehabil. 2006 Nov;33(11):827-32.

[6]. Bindl A, Mörmann WH. Marginal and internal fit of all-ceramic CAD/CAM crown-copings on chamfer preparations. J Oral Rehabil. 2005 Jun;32(6):441-7.

[7]. Quintas AF, Oliveira F, Bottino MA. Vertical marginal discrepancy of ceramic copings with different ceramic materials, finish lines, and luting agents: an in vitro evaluation. J Prosthet Dent. 2004 Sep;92(3):250-7.

[8]. Suárez MJ, González de Villaumbrosia P, Pradíes G, Lozano JF. Comparison of the marginal fit of Procera AllCeram crowns with two finish lines. Int J Prosthodont. 2003 May-Jun;16(3):229-32.

[9]. Nakamura T, Nonaka M, Maruyama T. In vitro fitting accuracy of copy-milled alumina cores and all-ceramic crowns. Int J Prosthodont. 2000 May-Jun;13(3):189-93.

[10]. Beschnidt SM, Strub JR. Evaluation of the marginal accuracy of different all-ceramic crown systems after simulation in the artificial mouth. J Oral Rehabil. 1999 Jul;26(7):582-93.

[11]. Rinke S, Hüls A, Jahn L. Marginal accuracy and fracture strength of conventional and copy-milled all-ceramic crowns. Int J Prosthodont. 1995 Jul-Aug;8(4):303-10.

[12]. Farid.F,Hajimiragha.H,Jelodar.R,MostafaviA.S,,Nokhbatolfoghahaie,H. In vitro evaluation of the effect of core thickness and fabrication stages on the marginal accuracy of all -ceramic system. Journal of Dentistry .2012:Vo19,No.3

[13]. Matty F.Abbate,Anthony H.L.Tjan,Wills M.Fox. Comparison of themarginal fit of various ceramic crown systems. J.Prosthet Dent 1989:61:527-31

[14]. Frankie Sulaiman, John Chai, Lee M. Jameson, Wayne T.Wozniak. A Comparison of the Marginal Fit of InCeram,IPS Empress and Procera Crowns. Int J.Prosthodont 1997:10:478-484

[15]. Kyu-Bok Lee,Charn-Woon PARK,Kyo-Han KIM and Tae-Yub KWON. Marginal and Internal Fit of All -ceramic Crowns Fabricated with Two Different CAD/CAM Systems. Dental Materials Journal 2008:27(3):422-426

[16]. Paolo Cardelli,Roberto Scotti ,Carlo Monacossss. Clinical fitting of CAD/CAM zirconia single crowns generated fron digital intraoral impressions based on active wavefront sampling. Journal of Dentistry 2011:10:1-7 